\title{
Speech To Speech Translation: Challenges and Future
}

\author{
Sandeep Dhawan \\ Senior IT Director
}

\begin{abstract}
The different languages in the world and the various native mother tongues have distinct origins. Speech is the most widely recognized method of articulation, with an average individual talking more than 11000 words each day.

Speech can make each other understand actions and thoughts, be it a dialogue, conversation, or even a presentation. The passage of information would be ineffective if the other party did not understand the language of communication. As a result, a system capable of bridging the linguistic divide is required. Speech-to-speech translation is one such system that can be useful in facilitating communication between people who speak different languages.

Efforts are constantly taken all around the globe to accomplish this objective and set it up as a regular occurrence to assist everybody. This paper describes a significant international and inter-institutional effort in this direction, highlighting the current challenges being faced as well as the future of the technology of Speech-to speech translation. The developed language-specific technology, parallel corpora, and speech unit (segmental) database are all been described.
\end{abstract}

Keywords: Speech-to-speech translation, languages, technology.

\section{INTRODUCTION}

The present circumstances and situations in the world demand the need for communication among speakers of various languages. SPEECH-TO-SPEECH translation (S2ST) is a human pipe dream that allows communication between people speaking different languages. The importance of S2ST technology is growing by the day because the world is becoming more borderless by the day. Moreso, the need for the exchange of information is inevitable because of the global and borderless economy, and speech is one of the means of achieving such. The borderless economy in the world has made it critical for speakers of different languages to be able to communicate. Speech translation which has been named as one of the top ten technologies that will transform the world, has long been a human dream and the ultimate aim is to improve communication between people who speak different languages. Speech-to-Speech Translation (SST) research topic represents a relatively recent research area in the Human Language Technologies arena, which helps in producing a speech signal in the target language that conveys the linguistic information contained in the source language's speech signal [1].

All of the languages that exist in our world have distinct origins, as does the set of their native mother tongue. According to the study Karunesh et al., 2013, learning a new language as an "adult" is more complex and expensive than learning as a child. Due to geographical factors, almost all of the world's population finds learning foreign languages extremely difficult. As a result, speech-to-speech translation technology would be a massive boon to everyone. Manual Translation of Speech to Speech has only been used for important official documents, news items, and award-winning literary works. There is a massive backlog of materials that need to be translated for administration, education, commerce, tourism, and other purposes. It is critical to have technical assistance in the form of machine translation aids. The rapid increase in demand for translingual conversations, spurred by 
Information Technologies and an increase in the number of borderless communities as evidenced by the increased number of EU countries, has increased the interest in S2ST research activities. The goal of speech-to-speech translation is to convert speech input from one language into speech in another. The technology facilitates communication between two or more people who speak different languages and can provide access to multimedia content in multiple languages $[1]$.

Speech to speech translation is a technology that converts spoken language into another language's speech. S2ST is significant because it allows speakers of various languages from around the world to communicate with one another, eradicating the language divide in global business and crosscultural exchange. It has been a colossal logical, social, and financial worth to humankind. One of the ten advances recorded in the article "10 Emerging Technologies That Will Change Your World" in the February 2004 issue of A MIT Enterprise Technology Review is "All-inclusive Translation." The piece features an assortment of interpretation innovations, with attention to speech translation technology.

Speech translation is a cycle that takes the conversational speech expressed in one language as info and deciphered speech phrases in one more language as a result. The three parts of speech-to-speech Translation are associated with a successive request. Automatic speech translation technology comprises of three separate advances: innovation to perceive (speech acknowledgment), innovation to decipher the perceived words (language interpretation), and innovation to integrate speech in the other individual's language (speech union). Late mechanical advances have made programmed interpretation of informal communication in Japanese, English, and Chinese for voyagers functional, and sequential interpretation of short, straightforward, conversational sentences spoken each, in turn, has become conceivable. Speech-to-Speech translation is a three-stage programming process that incorporates Automatic Speech Recognition, Machine Interpretation, and voice synthesis. ASR is liable for changing the communicated in expressions of source language over to the text in a similar language followed by machine interpretation which deciphers the source language close to the target language text. Lastly, the speech synthesizer is liable for the text-to-speech transformation of the target language [3]..

Speech-translation technology is essential because it allows speakers of many languages from all over the world to impart, connecting the language hole in worldwide business and cross-cultural communication. Speech translation would be highly beneficial to humanity in terms of science, culture, and economics. "Universal Translation" is one of the ten emerging technologies listed in the article "10 Emerging Technologies That Will Change Your World" in the issue of An MIT Enterprise Technology Review. The Universal Speech Translation Advanced Research Consortium (USTAR) is an international research collaboration formed to develop a network-based speech to speech translation (S2ST) with the goal of breaking down language barriers and implementing vocal communication between different languages around the world. The ITU-T standardized and approved international communication protocols in 2010, thanks to a U-STAR initiative.; Recommendations F.745 [4], and H.625 [5], enabling speech-to-speech translation (S2ST) modules to be connected across the globe over networks.

Multilingual speech-to-speech translation technologies are critical for bridging the language divide, which is one of globalization's most vital issues. Machine translation is the leading technology used in S2ST to generate natural translations from raw data. As a result, S2ST's performance is significantly reliant on the machine translation system's performance.

The purpose of automatic voice-to-speech translation is to produce a speech signal in one (target) language that transmits the linguistic information contained in another (source) language's speech signal.

In the subject of human language technology, significant progress has been accomplished. Various tasks, such as continuous speech recognition with an extensive vocabulary, speaker and language identification, spoken information 
International Journal of Computer Applications Technology and Research

Volume 11-Issue 03, 36-55, 2022, ISSN:-2319-8656

DOI:10.7753/IJCATR1103.1001

inquiry, information extraction, and cross-language retrieval in restricted domains, are now possible, and various prototypes and systems are in use. The difficulty of spoken Translation, on the other hand, remains a considerable challenge: "It was difficult enough to get a good text translation. It was more than traveling to the Moon for Speech to Speech MT - it was going to Mars..." [Steve Silbermann, Wired Magazine].

Speech translation (ST), the assignment of making an interpretation of acoustic speech signals into text in an unknown language, is a complex and diverse undertaking that expands upon work in automatic speech recognition (ASR) and machine translation (MT) [7].

\subsection{Applications:}

ST applications are diverse and include travel assistants, simultaneous lecture translation, movie dubbing/subtitling, language documentation, and crisis response, and developmental efforts [8].

Speech to speech translation is crucial for understanding cross-lingual spoken conversations and lectures and has been used in situations such as international travel and conferences. Existing speech to speech translation systems either use target text as a pivot (translating source speech into target text and then synthesizing target speech based on the translated text or directly translate source speech into target speech. The text matching to the target speech is used as pivots or supplemental training data in these translation systems [9]; otherwise, the
Translation would not be possible, or the translation accuracy would drop dramatically [9]

\subsection{Essential Features of the S2S Translation System}

A speech recognition system should be able to recognize speech in loud situations and from people who talk in a variety of ways. Machine translation systems must be domainagnostic and capable of translating a wide range of topics. Speech synthesis must achieve a more natural and expressive quality of speech. To develop the S2ST system, all of the researchers in this project-including speech processing and natural language researchers - are working together and closely. The speech recognition system must identify speakerindependent, ongoing, spontaneous conversational speech for the S2ST system to be successful [9] 10].

The centralized connections considered in this task are depicted in Figure 6. This will normalize the connection points and information organizations of the discourse interpretation engineering's modules, permitting them to convey over the Web. The data formats and interfaces of the modules of the speech translation architecture will be standardized as a result, allowing them to communicate over the Internet. It's also required to assemble standardized multilingual corpora and establish common speech recognition and translation dictionaries. Web-based HTTP 1.1 communication will be the primary communication interface, with a markup language called STML (speech translation markup language) being created as the data format for linking apps [2]. 


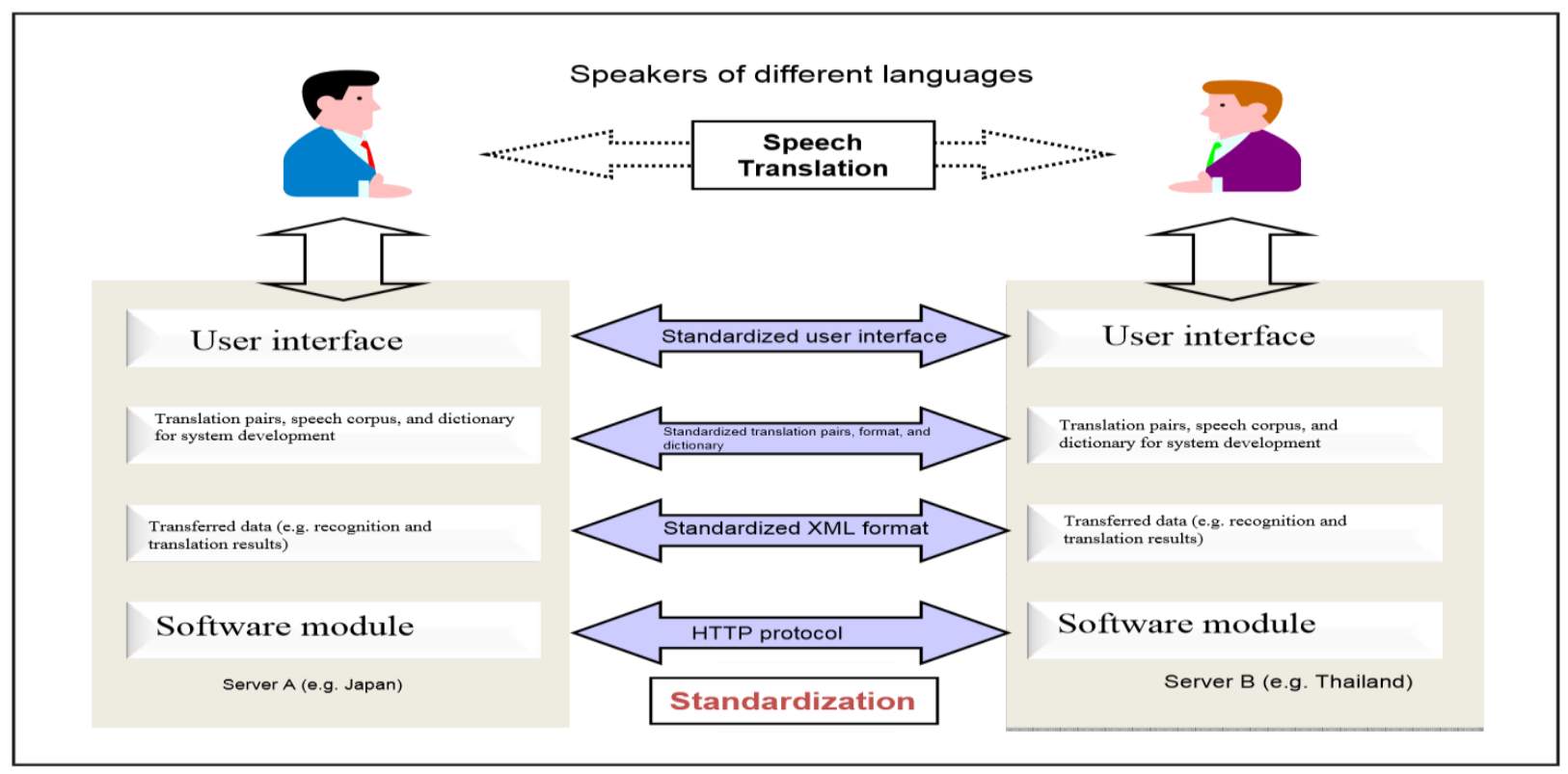

Figure 6 : Illustration of speech translation standardization

Source : Reference ${ }^{[12]}$

\subsection{History and world scenario of S2S translation systems}

Several previous studies have looked into the unsupervised conversion of speech to its corresponding phonetic categories (discrete tokens), which mimics how newborn children gain knowledge acoustic models in their mother tongue during their early years of life. (some of them only focus on a much easier task such as speech-to-text Translation. Among these works, vector quantized variational autoencoder (VQ-VAE) has been widely adopted and shown advantages over other methods. However, VQ-VAE is still purely unsupervised and cannot ensure the quality of the learned discrete representations. Therefore, although VQ-VAE performs very well on relatively more straightforward tasks like speech synthesis (Dunbar et al. 2019), it cannot achieve good accuracy on more complicated speech to speech translation where semantic representations of speech are necessary and more accurate phonetic words are required. Few works tackle speech to speech Translation for unwritten languages since it is incredibly challenging. [1,2, 3]
When NEC Corporation presented a display of speech translation as a conceptual demonstration at the 1983 ITU Telecom World (Telecom' 83), it drew a lot of interest. Realizing that speech translation will demand several decades of basic study, the Advanced Telecommunications Explore Institute International (ATR) was created in 1986 and launched a mission to study the subject. This initiative drew researchers from a wide spectrum of Japanese and foreign academic institutes [1]. In 1993, the ATR, Carnegie Melon University (CMU), and Siemens collaborated on a voice translation project that involved three locations around the world: the ATR, CMU, and Siemens. Speech translation initiatives were established all around the world after ATR's effort began. The Verbmobil plan was initiated in Germany, the Nespole and TC-Star programs have been established in the European Union, and the Transpac and GALE projects were launched in the United States. The GALE project began in 2006 with the goal of mechanically translating Arabic and Chinese into English. The aim of this assignment is to automate the extraction of critical multilingual information, which was previously done by humans; the project design is a batch text-output system. The ATR and NEC, on the other 
hand, aim to provide real-time voice translation for face-toface and non-face-to-face cross-language communication. Online speech-to-speech Translation is thus an integral component of this Research, and immediacy of processing is a key factor $[2,3]$.

The first time speech translation was observed was during the 1983 ITU Telecom World (Telecom'83), when NEC Corporation demonstrated it as a proof of concept. In 1993, the ATR, Carnegie Melon University (CMU), and Siemens collaborated on a speech translation experiment that involved three locations around the world: the ATR, CMU, and Siemens. The Verbmobil project was launched in Germany, the Nespole! and TC-Star projects were launched in the
European Union, and the Transpac and GALE projects were launched in the United States. The Translation of Research and development has developed from relatively simple to more complicated Translation. There have been a number of attempts to construct S2S Translation systems, with some success stories. The significant works in S2S Translation are shown in Table 1 in the annexure. [1,3]

The history of speech-translation technology is shown in Table 1. From meeting scheduling to hotel bookings to travel discussion, Research and development have steadily developed from relatively simple to more complicated Translation. Nevertheless, in the future, the supported fields will need to be expanded to allow a wider variety of basic and complex commercial interactions

Table 1: Trends in the Research and Development of Speech Translation

\begin{tabular}{|c|c|c|c|}
\hline Research Phase & $\begin{array}{l}\text { 1980s } \\
\text { Confirmation of Feasibility }\end{array}$ & $\begin{array}{l}\text { 1990s } \\
\text { Extension of Technology }\end{array}$ & $\begin{array}{l}\text { 2000s } \\
\text { Attempts at Practical Systems }\end{array}$ \\
\hline Fields & $\begin{array}{l}\text { Simple reservations (ATR- } \\
\text { phase 1) }\end{array}$ & $\begin{array}{l}\text { Reservations and scheduling } \\
\text { (ATR-phase 2, Verbmobil) }\end{array}$ & $\begin{array}{l}\text { *Everyday travel } \\
\text { conversation (ATR-phase 3) } \\
\text { *Translation of keynote } \\
\text { speeches (TC-Star) } \\
\text { *Conversation for military } \\
\text { use (TranTac) } \\
\text { *Intelligence collection (Gale) }\end{array}$ \\
\hline Linguistic features & $\begin{array}{l}\text { Expressions that are } \\
\text { grammatically accurate }\end{array}$ & $\begin{array}{l}\text { Everyday idioms that may or } \\
\text { may not be grammatical or } \\
\text { context-dependent }\end{array}$ & $\begin{array}{l}\text { Topics and proper nouns are } \\
\text { included in the expressions. }\end{array}$ \\
\hline Phonological features & Pronounced correctly & Pronunciation is ambiguous. & $\begin{array}{l}\text { Background noise is included } \\
\text { in the audio. }\end{array}$ \\
\hline Translation method & $\begin{array}{l}\text { Translation based on rules. } \\
\text { Artificial intermediate } \\
\text { language translation }\end{array}$ & $\begin{array}{l}\text { Translation based on } \\
\text { examples English is used as } \\
\text { an intermediary language in } \\
\text { the translation. }\end{array}$ & $\begin{array}{l}\text { Translation based on statistics } \\
\text { Multiple languages are directly } \\
\text { translated. }\end{array}$ \\
\hline
\end{tabular}

Recent improvements in speech translation technology have contributed significantly to the achievement of autonomous speech translation technology, which is among the three aspects of speech translation. Speech translation technology has a lengthy history dating over half a century. In 1946, immediately after the development of the very first computer.
Warren Weaver of the Rockefeller Foundation urged for studies into automatic translation technology. In the first place, the Rockefeller Foundation had a substantial impact on US scientific and technology policy. Then, in 1953, Georgetown University and IBM collaborated on automatic translation research using the 701 computers (the first commercial computer developed by IBM). This computer was 


\section{International Journal of Computer Applications Technology and Research \\ Volume 11-Issue 03, 36-55, 2022, ISSN:-2319-8656 \\ DOI:10.7753/IJCATR1103.1001}

used to create the world's first automatic translation system in 1954, proving the feasibility of translating from Russian to English. The translation capabilities of this system, which consisted of a lexicon of 250 phrases and six rules, were extremely restricted, but the presentation had a major influence on society. The linguistic barrier, it was thought at the time, would be broken relatively shortly. Following that, as a reaction to the shock of Sputnik's launch, the US government-funded an astounding \$20 million on automatic translation research. $[1,2]$

ALPAC delivered a somber report to the National Academy of Sciences in the United States in 1965. Because automatic Translation will not be practicable for the years ahead, the paper recommends that extensive Research be focused on language theory and comprehension to operate as the technology's foundations. Budgets for machine translation were subsequently curtailed in the United States, and the focus shifted to fundamental Research, with interpretation and comprehension as the key ideas. Winograd's language comprehension utilizing global knowledge in 1970 is a wellknown result from this period. However, the information basis for this type of study was insufficient and therefore cannot be considered to have positively linked with increased automatic translation performance in a broad or realistic sense.

In the early 1980s, Japan saw three major technology waves: rule-based Translation, example-based Translation, and statistically-based Translation. The $\mathrm{Mu}$ project, which intended to interpret abstracts from the Scientific and Technology Agency's science and technology literature, was a breakthrough in Japan. As a result, Research \& innovation into lexicon and guidelines (analytic grammar rules, conversion rules, and generative grammar rules) for rule-based machine translation managed to receive traction. Bravis, a company, has begun selling a commercial translation tool. This prompted big-name IT businesses, including Fujitsu, Toshiba, NEC, and Oki Electric Industry, to commercialize automatictranslation software. This rule-based technology is used in practically all commercial software packages today, as well as nearly all Web-based applications. Although improved and more thorough specialized lexicon was an efficient strategy to enhance translation quality, gradual but constant attempts to increase dictionary sizes beyond a few tens of thousands to millions of entries have been made.

Meanwhile, in 1981, Kyoto University professor Makoto Nagao drew inspiration from human Translation to design an example-based translation approach that uses phrases identical to the input text and their translations (together known as "example-based translations"). This example-based Translation joined with other Research at Kyoto University and ATR around 1990, spawned a second wave that swept the globe. Some commercialized rule-based systems have included this strategy; It is already being utilized as the foundation for a Japanese-to-Chinese technical and scientific publications translation initiative conducted by the National Institute of Information and Communications Technology (NICT). [5].

Later, in 1988, IBM presented an approach known as statistical machine translation, which combines a bilingual corpus with pure statistical processing that removes grammatical and other expertise. For a long time, nevertheless, this strategy received relatively little attention for a variety of reasons: the paper was tough to comprehend, the operating system was lacking, translation corpora were too minimal, the execution method was only published in patent specifications, and it was ineffective for languages other than related languages such as English and French. However, in the year 2000, a new approach known as phrase-based statistical machine translation was devised, and this ushered in the third significant wave, which was aided by larger bilingual corpora and more powerful computers.

ATR created its speech-translation system [1,2] by assembling a corpus of commonly spoken travel discussions is designed to enable travel conversation speech translation. The Research has so far produced a Basic Travel Expression Corpus (BTEC) with 1,000,000 matched pairs of Japanese and English sentences, as well as 500,000 matched pairs of JapaneseChinese and Japanese-Korean utterances. This is the world's biggest multilingual travel conversation translation corpus. The average length of the English sentences in the corpus is seven words, and they include subjects including greetings, 


\section{International Journal of Computer Applications Technology and Research \\ Volume 11-Issue 03, 36-55, 2022, ISSN:-2319-8656 \\ DOI:10.7753/IJCATR1103.1001}

difficulties, purchasing, transit, hotel, tourism, meals, communication, airports, and business. Computers with increased processing power and memory, as well as more pervasive networks, are allowing portable speech translation systems to be implemented. Developments are taking place in the establishment of standalone systems on small hardware as well as distributed implementations that connect mobile phones and other devices to high-performance servers across the network. Due to concerns such as size, weight, and battery life, the standalone technique is not possible to apply on a portable computer. In instances when wifi and other facilities are unavailable, there is also anticipated to be a demand. As a result of these concerns, attempts are made to commercialize specialized mobile devices with built-in speech-translation capabilities. NEC produced the earliest commercial mobile device with onboard Japanese-to-English voice translation in 2006 (with hardware requirements of a 400-MHz MPU and 64 MB of RAM). Meanwhile, in November 2007, ATR created a distributed version of a speech translation system for the DoCoMo 905 i family of mobile phones utilizing mobile phones and network servers. The technique, termed "shabette honyaku" (see Figure 4), was developed by ATR-Trek and is the world's first mobile phone-based speech translation service. Then, in May 2008, the DoCoMo 906i series launched a Japanese-to-Chinese speech-translation service. The design of the speech recognition module utilized in distributed speech translation is shown in Figure 5.[2].

Background noise reduction, acoustic analysis, and ETSIES 202 050-compliant encoding are all performed on the mobile device (front end), and only the bitstream data is sent to the speech recognition server. The back end of the speech recognition server extends the incoming bitstream, conducts speech recognition, and determines word reliability. One of the advantages of this design process is that it is not constrained by the mobile phone's information-processing limits, allowing for the deployment of large-scale, extremely exact phonological and linguistic models. Because these models are stored on a server rather than a mobile phone, they are simple to update, allowing them to be kept up to current at all times. The technology is already in widespread use: as of June 2008 , it has been used by a total of $5,000,000$ people. [1, 2].

\subsection{The basic model for Speech-to- Speech Translation}

The high-tech Speech-to-Speech Translation system that empowers such multilingual communications supports a pipelined architecture of automatic speech recognition, machine translation, and speech synthesis or text-to-speech that mainly depends on linguistic information while disregarding some other valuable insights existing in speech and spoken discourse such as noise and human utterances. There is minimal connection between the fundamental components prior to the pipeline, and no people are involved in the loop for autonomous learning, adaptation, or collectively controlling the interaction. To transcend these fundamental problems, pipeline resilience must be built at all levels.

The system's foundation is that it should take advantage of the rich context that exists outside of the dictated words while also being aware of and cooperating with various cultures to improve information transfer, communication efficiency, and social co-presence in order to enable successful multilingual interactions. Various projects in this study capture that model and transmit highly linked information for voice prosody, discourse, and user state behavior in order to allow robust translation and considerable synthesis of the target language [3]. 


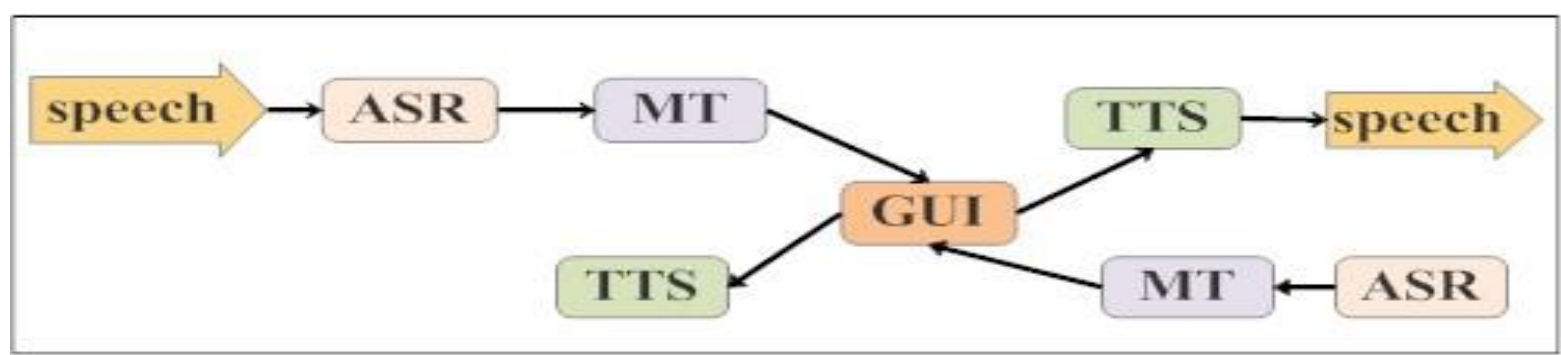

Figure 1: Overall Speech-to-Speech translation system

\section{CHALlengeS}

Computer-assisted cross-lingual conversation by automatic speech-to-speech Translation has been one of the most challenging problems in spoken language technologies in decades. Recent remarkable advances in speech and language processing led by deep learning techniques benefit this challenge by real-time and accurate speech translation. One crucial problem in automatic speech-to-speech Translation is its delay. Spoken language processing tasks are usually handled at the utterance or sentence level. Their application to speech-to-speech Translation suffers from a long delay that is proportional to the input length because the process starts after the observation of the end of an utterance. That is similar to consecutive interpretation and is not useful for long monologues such as lecture talks. On the other hand, in such situations, simultaneous interpretation is often used for an audience not proficient in the language of a talk. Simultaneous interpretation is a challenging task to listen to the talk and speak its interpretation in a different language.

Attributed to the reason that state-of-the-art automatic speech recognition and machine translation systems are far from flawless, there are substantial outstanding research concerns that impede the implementation of normal and unrestrained voice-to-speech translation systems, even for relatively limited application domains. Furthermore, unlike interpreting written information, colloquial spoken words are frequently communicated with sloppy grammar and informal, spontaneous speech [Ref 1.2].

In fact, while creating demonstration systems, substantial limits on the application domain and the sort and format of allowable utterances are often imposed, i. e., both in terms of the range and breadth of user input that can be provided at any time during the encounter. As a result, the system's flexibility and naturalness of use are jeopardized.

S2ST between Western languages and non-Western languages, such as English-from/to-Japanese or Englishfrom/to-Chinese, necessitates the use of technologies to overcome the linguistic differences. A translation from Japanese to English, for example, necessitates 1) a word separation process for Japanese due to the lack of explicit spacing information, and 2) transforming the source sentence into a target sentence with a drastically different style due to their word order and word coverage, among other factors. Another factor for S2ST is that the technology must be portable across all domains because S2ST systems are frequently utilized for applications in specialized situations, such as facilitating nonnative language discussions by tourists. As a result, the $\mathrm{S} 2 \mathrm{ST}$ technique in voice recognition, machine translation, and speech synthesis must incorporate (semi)automated mechanisms for adapting to unique situations/domains and language pairs [3].

Some other challenges in exploiting rich contextual features in machine-mediated speech-to-speech Translation include:

1. Designing appropriate user interfaces that can augment the translation hypotheses with contextual information.

2. Detecting and exploiting source language prosody and dialog information in target text-to-speech synthesis.

3. Actively learning and adapting the system from a user feedback or user in the loop. 


\section{International Journal of Computer Applications Technology and Research \\ Volume 11-Issue 03, 36-55, 2022, ISSN:-2319-8656 \\ DOI:10.7753/IJCATR1103.1001}

However, there are indeed numerous obstacles to overcome before this technology becomes viable. The requirement to encourage new languages, as well as the automatic adoption of town names, given names, and other common words, are only a few examples. Simultaneous interpretation, in which a continuous stream of speech is interpreted, should also be developed using technology. Speech translation systems have a wide range of applications, including speech information retrieval, interactive navigation, dictation, summarization, and archiving, and more applications are predicted to arise.

S2S technology's three aspects (speech recognition, language translation, and speech synthesis) each have their own set of challenges. This innovation requires the recognition and Translation of spoken language, which is far more challenging than interpreting text since spoken language comprises ungrammatical, conversational idioms and lacks punctuation such as question marks, exclamation marks, and quote marks. Speech recognition errors also lead to significant mistranslations. As a result, instead of enabling all types of discussion from the start, researchers have selected a development approach that focuses on boosting accuracy to a useable level by firstly constraining the system to comparatively straightforward speech [Ref 1.2].

An instance of spoken English translations of a Japanese statement can be found underneath. "Mado o akete mo ii desu ka" means "mado o akete mo ii desu ka" in Japanese. Here are the English sentences that correspond:

1. Is it okay if I leave the lid open? 2. Is it alright if I open the curtains? 3. Is it possible for me to open the window? 4. could we breach the window 5 . is it alright if I open the window 6 . would you mind if I rolled down the window 7. is it alright if I open the window 8. do you mind if I open the window 9. would it be alright if I open the window 10.

As all these examples demonstrate, speech translation utterances are not entire statements - they frequently lack subjects, and no capitalization is utilized in subjects and proper nouns - and perhaps even questions lack question marks. Highly conversational terms must also be dealt with. A dataset called Field Experiment Data was also analyzed, and so was information obtained from a corpus of roughly 10,000 utterances of dialog captured under real-life situations and mediated by a speech translation system called Machine Aided Data (MAD) (FED). This information was obtained over the course of five days at Kansai International Airport, with the assistance of the Osaka Prefecture, throughout December 2004 and January 2005. The information comprises approximately 2,000 utterances of discussion handled by a speech-translation system involving foreign-language speakers (39 English speakers and 36 Chinese speakers) and facilitators at a tourist resort [4].

However, there are still numerous research hurdles to overcome; specifically, there is a high level of speaker reliance and expression variability; also, new terms and ideas are continuously being developed in response to societal changes. Speech translation technology is now limited to short utterances of roughly seven words, such as a trip chat. As a result, there are many other unanswered questions before speech translation can handle long, complex speeches like those seen in newspapers or lectures. Some of the immediate technical challenges include:

1. Standardization for using the technology to connect speech translators all around the world.

2. Monitoring and assessing usability in practical applications.

3. Relaxation of copyright to allow web usage of example translations

4. Using the most recent proper nouns based on the user's present location

5. Multiple language support is available.

There are thousands of unwritten languages in the world, which are purely spoken and have no written text. Just like in Jia et al. (2019), prolonged speech (which generally includes information, context, communicating style, and other factors) is far more adaptable than discrete symbols in representing semantic meanings (text), this renders speech translation more difficult than text translation. As a result, reducing the continuous fluid space of speech into a more constrained 
discrete area is the best way of making speech translation easier for unwritten languages.

The creation of S2S systems necessitates the collection of diverse types and quantities of spoken language data: several hours of acoustic speech data, adequately reflecting dialectal variants, for language modeling, there are several hundred thousand active words of domain data, as well as millions of words of parallel text in target language pairings for machine translation.

\section{LISTS OF CHALLENGES}

\subsection{Existing and collected data}

The initial barrier in both Pashto and Persian was indeed the lack of speech/language input in any form - lexicon, translations, or acoustic data. The First Discourse data set, which contains 20 read sentences from 300 individuals going in age, sex, schooling level, and tongue, for a sum of 6000 expressions, was the sole information accessible on the Persian side (available from ELDA). Because the transcripts were unsuitable for voice recognition, they had to be reconstructed. To supplement these transcripts, we enlisted the help of Persian speakers from all across Los Angeles, who provided read and semi-spontaneous speech data. In a Wizard of Oz-style scenario, the semi-spontaneous speech was elicited, while read speech was gathered and confirmed by the speakers themselves using an interactive data collection tool. These data couldn't be used to simulate language: Parallel to creating the data mining approaches mentioned below, 300 Standardized Patient (medical student-actor patient) sessions were conducted at the USC campus in partnership with the Medical School to gather data for language models [1]. Following that, the SP data was transcribed, yielding almost 300,000 words of in-domain data in both English and Persian. The only pre-existing corpus of recorded Pashto we could uncover was a series of untranscribed Voice of America (VOA) Pashto service broadcasts, which the Linguistic Data Consortium had captured from the broadcasts. The data, on the other hand, were not well suited for the task: the broadcasts were controlled by less than twelve speakers, exhibited just a proportion of Pashto dialect variety, were of poor audio quality, and did not represent dialogue speech pattern. As a result, we gathered about 80 Pashtuns from a nearby emigre population and asked each of them to record 100-200 randomly created utterances, including responses to inquiries. The entire amount of speech recorded was around 7 hours. The VOA data was also translated for 5 hours. These 12 hours of speech, or nearly 100,000 running words, were used to develop the acoustic model.

\subsection{Transcriptions \& Lexicons}

The transcription schemes of the languages, particularly those that can enable machine spoken language processing, have been the singular biggest impediment to all parts of the Pashto and Persian languages in this work. Because Pashto lacks a uniform, standard system of writing or spelling rules, a single word can be written in a variety of ways, and distinct words can be spelled in the same way. We attempted to translate acoustic data straight into a phonemic description to avoid the non-standardization of the original orthography. However, we discovered that translators struggled to recognize the phonemes, which was made even more complicated by the fact that our phonology assessment was designed to encompass a wide variety of Pashto dialects. As a result, we switched to indigenous (Arabic-based) writing, which, despite its challenges, proved to be more trustworthy. The set of words was, therefore, phonemically translated in indigenous script orthography. Each script format was often connected with several phonemic representations. These representations could indicate significantly different pronunciations, totally different words written in just the same style, or syntactic patterns that are distinct but equivalent. Similarly, any given phonemic representation can be connected with one or more script forms, each with one or more intended meanings. Such texts might be used for language modeling speech recognition processing using an isomorphism of the indigenous script. This isomorphism was creating ambiguity both at the input and at the output of the recognizer. Each "isomorphic class," which served as a "word" for all practical purposes of recognizer training and testing, had many pronunciations, sometimes quite a few, affecting acoustic model training and 


\section{International Journal of Computer Applications Technology and Research \\ Volume 11-Issue 03, 36-55, 2022, ISSN:-2319-8656 \\ DOI:10.7753/IJCATR1103.1001}

search accuracy. The recognizer's output, on the other hand, matched to a series of word classes and did not distinguish between word meanings within the same class; therefore, it was handed to the translation engine to handle. Although Persian has a standardized writing system, it is not well-suited for use in a speech-to-speech translation system. The Modern Persian alphabet is based on the Arabic alphabet, with the addition of four letters and changes to two-character designs. Despite the capability to indicate vowel sounds in the written script, this process is rarely used in Persian transcription, resulting in a lossy encoding of the required orthography (this problem incidentally is common, at varying degrees, to all languages that use the Arabic script). Three alternative encoding techniques were devised as a result of the solution: A one-to-one mapping from the Arabic script to the Latin alphabet (USCPers), an augmented version (USCPers+) that encodes the extra vowel information found in spoken Persian, and a phonetic transcription scheme (USCPron) that allows the ASR and TTS components to be created [3]. Given the structure of the foregoing transcription strategies, the very next step was to gather enough data to create a lexicon with mappings all along three dimensions. Clearly, generating pronunciation from the Arabic script is an ill-posed problem, necessitating the need of humans to create dictionaries. Transcribers and transliterators turned clean English utterances into these numerous formats during the first data collection, building pronunciation dictionaries at the same time. Because some early data was available, statistical learning methods might be used to automate the procedure.

\subsection{Acoustic modeling}

We employed front-ends with $16 \mathrm{kHz}$ sampling rate, $10 \mathrm{~ms}$ frame advance rate, 12 mel frequency cepstral coefficients plus normalized energy, and first- and second-order differences for both Persian and Pashto systems (39 features). Persian contains 34 phonetic units (29 phonemes, silence, br, ls, ga, and LG), whereas Pashto has 43. (41 phones, silence, reject). State clustering was used to train 3-state triphone hidden Markov models (HMMS). 4207 clustered states were utilized in the Persian, with an average of 14 Gaussians per state. To match the limits of the predicted tiny footprint platform, the Pashto system employed a substantially lower model size (129 phone-state Gaussian clusters with 32 Gaussians each, trained via discriminative maximum mutual information estimation) (MMIE, [5]).

The Persian system employed the SONIC [6] speech recognition engine, whereas the Pashto system used DYNASPEAK [7]. Both techniques employed an English phoneme mapping into the target language to initialize the models, which were then altered or reassigned using the limited amount of information available. For Pashto, we only employed a knowledge-based (linguistic) phone mapping approach; however, for Persian, we looked at three distinct methods: knowledge-based, data-driven phoneme mapping, and data-driven state mapping. The Earth Movers Distance (EMD) method was used in the data-driven strategies to try to reduce the amount of effort required to turn one GMM into another. We improved phoneme level recognition by 2.7 percent utilizing EMD at the sub-phoneme level, compared to using simply Persian speech data. This benefit, while appealing for little amounts of data, becomes unimportant as the quantity of the data grows. The models produced from cross-lingual phonetic alignment were exclusively employed for alignment reasons in that scenario. As we expand our translation system into languages with fewer resources, we want to take advantage of cross-lingual expertise even more. We currently have speech recognition engines in multiple languages (English, Persian, Arabic, Greek, and so on), so the pool of potential GMM mixtures is growing, and we anticipate that the potential benefits will be greater and that a procedure for rapid language portability into new languages and dialects will be developed.

\subsection{Language modeling}

To offer enough surface form coverage for these 2-way S2S systems, language modeling requires orders of magnitude more data than acoustic modeling. Written material can, however, be utilized as a rough approximation to spoken language transcripts, as is common practice (although easy 


\section{International Journal of Computer Applications Technology and Research \\ Volume 11-Issue 03, 36-55, 2022, ISSN:-2319-8656 \\ DOI:10.7753/IJCATR1103.1001}

access to text data may be difficult, e.g., Persian, or even not possible for some of the target languages, e.g., Pashto). We faced two key challenges in developing adequate language models for the Persian-English and Pashto-English translation systems. The first was a lack of (medical) domain data, and the second was a lack of generic background data for bootstrap in Persian and Pashto. Take the Persian-English system, for example, where there were some currently accessible textual materials (medical domain data in English and some Persian text). Multiple simultaneous procedures were used to generate relevant domain data: The initial stage was to find any existing medical domain text, which we accomplished through the use of medical phrasebooks, paraphrase, and Wizard of $\mathrm{Oz}$ data collectors, among other methods. This content is clearly restricted, which was used as a starting point for mining web data. The Web has a lot of text and even some transcribed information, but it's also tough to find and automatically filter the relevant in-domain content. Our first attempts were centered on a bag-of-words technique [8], but we eventually created far more powerful algorithms. The current approach [9] is centered on an iterative Web crawling strategy that employs a competitive set of adaptive models, including a generic topic, a noise framework indicating spurious text commonly faced in web-based data (Web data), and a topicspecific model to yield query strings for WWW search engines using a relative entropy-based approach and to weigh the available to download Web data appropriately for building topic-specific language models. When compared with the results of a generic model built with only $5 \mathrm{~K}$ words of indomain data as a seed corpus, this method yielded a 14 percent improvement.

In addition to supplying in-domain English data, we utilized the simplified bag-of-words web data technique to mine Persian text, allowing us to develop a Persian background language model. Caused by a lack of relevant Persian web resources, this initiative did not achieve the same level of success. In addition to establishing data mining tools, 300 Standardized Patient (medical student-actor patient) sessions were held on the USC campus in partnership with the Medical School. The SP data was then translated, yielding more than
300,000 words of in-domain data. The language models that resulted were built on layers of data, most notably medical phrasebooks and paraphrases (English and Persian) in domains that were personally gathered (generic web-data, domain web-data, and generic models).

Approximately 21,000 English words and more than 8000 Persian words are represented in the models. Moreover, both the English and Persian models are class-based, allowing us to supplement crucial classes such as prescription names, pleasantries, connections, and various sorts of lexical features with human knowledge. Language modeling was made considerably more difficult due to Pashto's morphological complexity and the limited amount of accessible training data. We solved the problem by adopting the technique described in and creating a language model with finer backoff layers than a standard word language model. To do so, we first created a vocabulary clustering tree, with the root representing the whole vocabulary and each node representing a node with all terms in its descendent nodes. The tree is created using a measure of similarity depending on the left and right contexts of a word and the least discriminative information clustering technique. When assessing the transition probabilities of a word based on its n-gram prefix, we first back off to its context, replacing the most distant word with its class, from the most specific to the most general, and then back off to the normal lower-order (n-1)-gram prefix if none of these backoffs could guarantee a minimum number of occurrences. The language model that results has a relative perplexity reduction of over $10 \%$ and a sig.

\subsection{Speech recognition}

Several speech recognition tasks have been explored and assessed during the last 15 years. Each task proffered its own set of difficulties. The following characteristics distinguish such tasks: kind of speech (pre-rehearsed vs. spontaneous), communication goal (computer, audience, person), and bandwidth (FWB, full bandwidth TWB, telephone bandwidth, FF, far-field). Dictation (WSJ), broadcast news, switchboard, voicemail, and meetings are just a few of the responsibilities. 
The following is a list of them in order of word error rate (wer) 7 percent dictation, well-formed, computer, FBW 12 percent, varied, viewer, broadcast media FBW Voicemail: 30 percent spontaneous, individual, TWB Switchboard: 20-30\% spontaneous, person, TWB Meetings are 50-60\% spontaneous, with person FF. At the moment, the trait having the greatest impact on word mistake rate is spontaneous speech, preceded by environmental influence and domain dependency.

\subsection{Speech synthesis}

In a voice-to-speech translation system, speech synthesis is essential. One of the most difficult goals for speech synthesis is to mimic the human voice. Gender, age, and cultural adaptation are all new challenges in the multilingual humanto-human communication framework. Emotion and prosody are also crucial considerations. [7] [8]. Concatenation of different acoustic units is currently the most effective way to generate synthetic speech. This method differs from previous rule-based synthesis, which requires explicit knowledge and competence to construct the deterministic units. Because the unit selection process in a corpus-based approach encompasses a combinational search across the whole speech corpus, fast search algorithms have been proposed as an integral part of the current synthesis process. The primary components of corpus-based approaches for specifying the speech segments necessary for concatenative synthesis are a unit selection algorithm, some objective measurements utilized in the selection criteria, and lastly, the development of the needed speech corpus. From an application standpoint, the massive amount of storage required to leverage the concatenation of speech units severely restricts the type of application. The other two major challenges in speech synthesis are prosody and speaker characteristics, as well as speech segment design. It is vital to provide sufficient tone and emphasis, rhythm, pace, and accent in order to manage prosody. There is a need for segmental duration control and fundamental frequency control. The global spectral features reflecting vocal tract characteristics, as well as the glottal waveform of voice excitation, contain not only language information but also speaker voice characteristics. Furthermore, as indicated by variations in voice quality and prosody, paralinguistic elements impact speaking styles. The majority of improvements are anticipated to come from prosodic modeling. Investigating this field and attempting to master the language and extralinguistic phenomena will almost certainly address multicultural issues, which are crucial in a multilingual communication process.

\subsection{Machine Translation}

Besides speech recognition and synthesis, the translation component is the core of a speech-to-speech translation system. The standard machine translation (MT) issue, which involves translating a text from a source language, such as Italian, into a target language, such as Chinese, is not the same as the S2PT problem. To begin with, no individual is engaged in the standard MT dilemma. The procedure is oneway only. The text is designed to be 'correct' linguistically. Two persons are participating in the S2ST process, the procedure is bi-directional, and the speech is colloquial, spontaneous, ungrammatical, and combined with non-verbal cues. Furthermore, the surroundings are a key concern in terms of acoustic noise and interaction mode. In S2ST, near-realtime Translation is required. Then, because people are effectively engaged in the process, the comprehending task is carried out collaboratively by individuals. Finally, because a machine is engaged in the interpretation in any case, there is a significant problem of human-machine communication to address. All of these elements must be considered in order to address the S2ST problem. Various designs were used, some of which use an intermediary language (interlingua, interchange format), and others use a direct translation approach. JANUS and NESPOLE architectures are two common examples of the first scenario. [9]. The analysis and synthesis chains are the two main processing chains in the Italian implementation of the NESPOLE [S2ST system architecture]. The analysis chain converts an Italian acoustic signal into a (sequence of) IF representation(s) by passing it through the recognizer, which also generates a succession of word hypotheses for the input signal; and the recognizing 
module, which delivers IF representations using a multi-layer argument extractor and a statistical-based classifier. The synthesis chain starts with an IF expression and ends with a synthesized audio message in the target language that expresses that content. It is made up of two modules. The generator transforms the IF representation into a more language-oriented model before combining it with the domain expertise to generate Italian phrases. These kinds of statements are sent into a voice synthesizer. The ATRMATRIX architecture is an exemplification of the direct translation approach [10], as it employs a cascade of a speech recognizer with a direct translation algorithm, TDMT, whose produced text is then synthesized. Example-based algorithms are used to accomplish the direct translation technique. Starting with text translation, IBM[11] [12] pioneered the second example of direct Translation based on statistical modeling. Statistical Translation has also been created as part of the EU-TRANS project and the VERBMOBIL project in Germany. Currently, Research is underway to develop unified or integrated techniques. The quintessential aim of this model is to harmonize speech recognition, understanding, and Translation as whole statistical processing. "For a future study on spoken language translation, we consider this integrated methodology and its appropriate implementation to be an open question." The most important experience gained in the VERBMOBIL project, in particular a large-scale end-to-end evaluation, revealed that the statistical approach resulted in significantly lower error rates than three competing Translation approaches: the sentence error rate was $29 \%$, compared to $52 \%$ to $62 \%$ for the other translation approaches. Furthermore, the end-to-end assessment process is a critical concern for S2ST systems. The focus is to create a strategy that is based on objective facts. VERBMOBIL, CSTAR, and a number of other organizations have suggested and created evaluation systems.

\subsection{Improve the end-to-end performance significantly.}

This is the main test to be tended to sooner rather than later. It appears to be that brought together techniques in light of factual displaying are exceptionally encouraging, given that a few central questions will be managed and appropriate arrangements worked out. This approach permits the incorporation of acoustics, phonetic setting, talking rate, speaker varieties, language highlights like sentence structure or semantics, and so forth into one bound together with the way. Then, at that point, this approach mutually streamlines acoustics, language, and speaker impacts. From the displaying point of you, it addresses, all in all, a shift from the source model. Significantly more work is required in proposing new computational instruments and developing them. This approach is likewise steady with the speech synthesis perspective: corpus-based and information-driven A test will likewise be the double-dealing of genuine applications in a restricted area, i.e., the travel industry, of frameworks in light of interlingua draws near. Central questions for this situation are movability and power.

\subsection{Produce aligned multilingual corpora and lexica}

Corpora and lexica are a vital problem in having to provide the difficulty of constructing new models in the hopes of greatly improving performance. There have been plans to gather and transcribe 5000 hours of spontaneous speech in order to solve the challenge of spontaneous speech recognition [14]. This is indeed a contentious issue, but it is what we have discovered from our previous speech recognition experience. The test data could come from both old and news sources. Multilingual text corpora that are aligned for Translation are also essential. A joint effort is underway with ATR and IRST, as well as the other members of the CSTAR III consortium, to create aligned text corpora composed of transcription and Translation of phrasebooks in the tourism domain. This phrasebook covers a broad range of situations: emergency, timetable, transport, sightseeing, directions, attractions, hotels, shopping. Aligned multilingual lexical are also important language resources for future S2ST systems development. Current activity is under development in LC-STAR [15], a newly funded project in the Vth framework by the EU. 


\subsubsection{Integrate speech to speech} translation components in real applications

Real services and applications involving speech communication need to manage the "interface problem," i.e., the physical impact of the user with a device that involves multimodal multimedia in a ubiquitous environment. A wearable device, a PDA or $3 \mathrm{G}$ cellular, cannot be operated by a keyboard and requires sophisticated natural multimodal human interfaces. Speech, vision, and handwriting seem natural candidates for human-machine interaction. But how can a system provide seamless integration between humanmachine services and human-human services? How can the system blend the two, provide assistance and guidance for a user to access and understand databases and information resources, but also serve as a go-between to facilitate the interaction with other humans or with a user's direct environment?

\section{A NEW ACTION IN EUROPE}

Given the challenges previously discussed and the experience carried on in the previous and ongoing projects, a new and innovative initiative is needed to tackle the problem. This initiative, in order to be successful, needs, first of all, a critical mass of researchers. Within Europe, few research groups have the capability to build up complete SST systems. Most research groups are small and work only on some research themes, i.e., prosody, acoustic modeling, language modeling, speech synthesis. Although these small groups may have excellent researchers, their work has less impact on the development of SST components. This new initiative should provide an appropriate infrastructure to use in an effective way the intellectual potential of European researchers. Given the big shift needed in order to set up this new action, a group of major European players in the spoken language technology, both research institutions, industrial entities, and ELDA, proposed a preparatory action, which acronym is TC-STAR_P (Technology and Corpora for speech translation).

\subsection{Goals and Activities}

The preparatory action, under negotiation, fits with the action line IST2002-III.5.2 c) "preparing for future research activities." It is scheduled to begin in July 2002. The duration will be one year with the purpose of preparing and getting ready an integrated project for the VI Framework. An integrated project is a large-scale action with the purpose of creating the European Research Area, ERA. The activity of the TC-STAR_P will be carried on by the cooperation of the four groups: an industrial group with proven experience in SST technology development, a research group with proven experience in Research in SST technologies, an infrastructure group with proven experience in producing language resources for SST components and with proven experience of evaluation of SST components and systems. Then a dissemination group will be in charge of using and spreading the project's results. Three are the main goals of this action: • developing research roadmaps and associated implementation models $\bullet$ identifying and bringing together all relevant actors in the Speech-to-Speech Translation (SST) area • investigating effective mechanisms for managing future activities 4.1.1 Preparing RTD roadmaps and associated implementation models The consortium is composed of different RTD communities: industrial, academics, and infrastructure entities. All these organizations will contribute to developing common visions and analyzing research requirements for SST systems. As a result of these tasks, industrial partners will prepare roadmaps for technical implementations and services; the scientific and academic groups will prepare roadmaps for technology improvements, and the infrastructure group will provide roadmaps for production and evaluation campaigns. The work will include a case study where industrial partners and research partners will provide application-oriented and Research input, respectively. The infrastructure group will focus on preparatory tasks for setting up production, evaluation, and validation centers for the needed LR. 4.1.2 Identifying and bringing together all relevant actors the consortium includes some of the most relevant actors in the SST field. One of the objectives during the lifetime of the project is to attract further key actors from the Industrial 


\section{International Journal of Computer Applications Technology and Research \\ Volume 11-Issue 03, 36-55, 2022, ISSN:-2319-8656 \\ DOI:10.7753/IJCATR1103.1001}

Research and infrastructure groups, as well as SMEs working with SST applications and related fields. Within the infrastructure group, the key action is to attract and prepare contacts with national agencies for funding language-specific LR-production in the future FP6 and with entities working on the evaluation and validation of language resources. The development of language resources is a very expensive activity, which must be best tackled by coordinated funding actions at national and European levels. 4.1.3 Investigating a new management model According to the IST 2002 Work program, Action Line 3.5.2 should focus on building and strengthening RTD communities by encouraging Research, business, and user organizations to develop together common visions and analyze research requirements in order to identify common challenges and objectives; and on investigating effective mechanisms for managing future activities. Moreover, a cornerstone of the future work to be developed under the Integrated Project is the management structure. In accordance with Action Line 3.5.2., the work to be performed under TCSTAR_P includes exploring a new organizational model in order to allow partners to collaborate in pursuing the final goal smoothly. This important task will be investigated during the project. Issues such as distribution of work and resources, admission and withdrawal of participants, engagement of additional parties, scientific guidance, monitoring, etc., will be examined. The model has to be effective to reach the envisaged goal, to react to external new trends, needs, and demands coming from the market, society, and scientific community. Section 2

\subsection{A Comparative Study with Human Speech-Translation Capability}

Essentially, evaluating the efficiency of speech translation is exceedingly challenging. If the speech synthesis component is not included in the assessment, the efficiency of the result is assessed by entering a number of sample sentences into the system. The approach for assessing voice translation is largely the same as the model for assessing computerized text translation in this regard. Speech translation, on the other hand, evaluates utterances rather than strings of text. To assess translation quality, two approaches are used: one in which the translations are humanly rated on a five-point scale, as well as another in which the closeness between both the system's output and previously generated reference translations are compared. For the latter, a range of evaluation scales has been suggested, including BLEU, NIST, and word error rate (WER). These scales have become increasingly popular in recent years. These data can be used to compare two different systems because they are basic numerical values. What these scores can't tell you is how well the higher-scoring system will function in the actual world. A strategy for resolving the situation has been presented, which involves measuring system performance in human terms and calculating the system's Test of English for International Communication (TOEIC) score. To begin, native speakers having documented TOEIC scores ("TOEIC takers") are requested to pay heed to test Japanese statements and interpret them into spoken English.

Following that, Japanese-English bilingual assessors evaluate the TOEIC takers' interpretations of the results of the speechtranslation system. The fraction of test phrases for which the humans' translations are better would then be measured as the human win rate. After calculating the human win rate for all TOEIC takers, regression analysis is utilized to generate the speech-translation system's TOEIC score.

Figure 2 depicts the system's performance in TOEIC scores. The speech-translation method is virtually always correct when employing relatively brief utterances like those in basic travel conversation (BTEC). However, the efficiency of the speech-translation system on conversational speech (MAD and FED) is comparable to a Japanese speaker's TOEIC score of 600 . Additionally, when coping with prolonged, infrequent, or complicated utterances, performance suffers dramatically. As a result, there is still potential for development in terms of performance. 


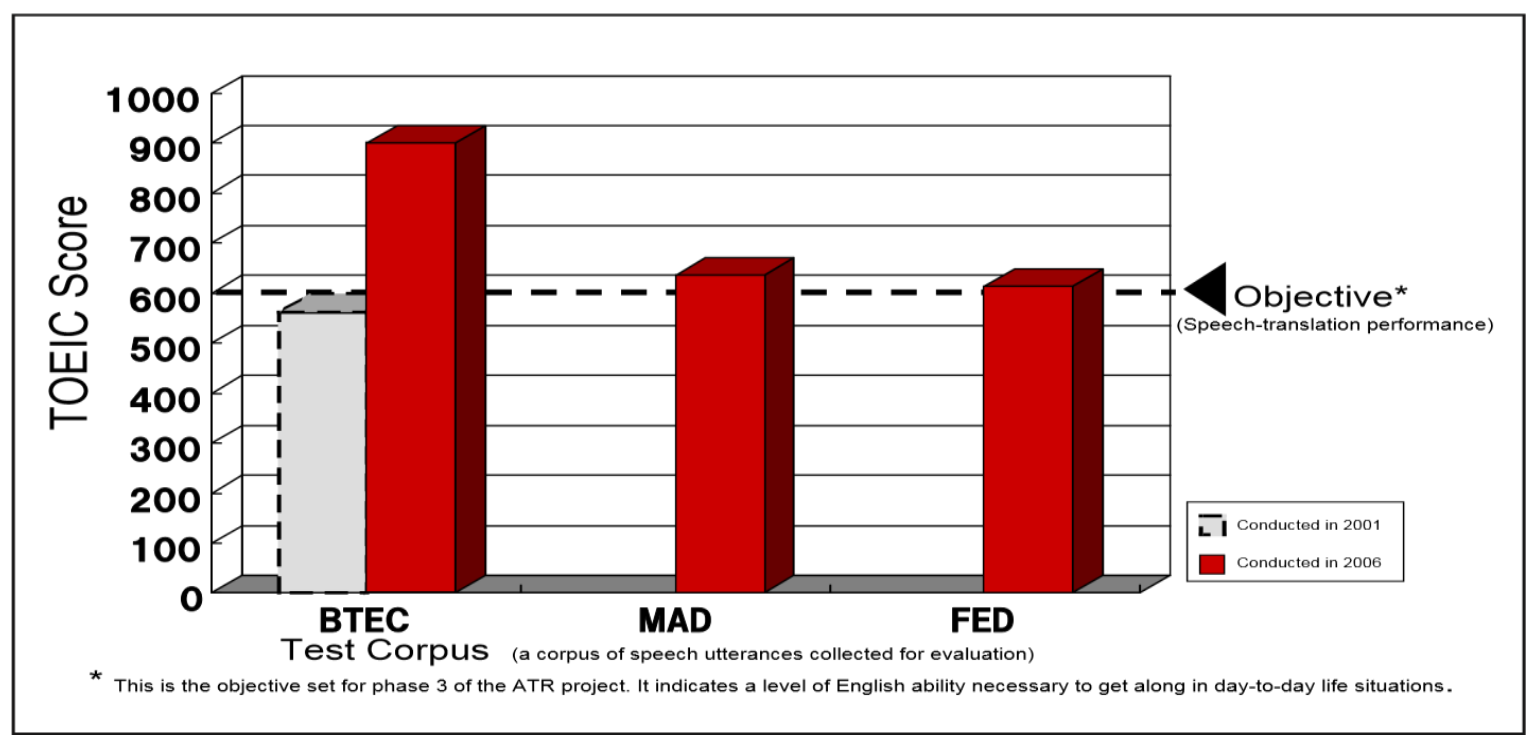

Figure 2: TOEIC scores are an instance of how to assess the reliability of speech translation.

Source (S.Nakamura et al., "ATR Multi-lingual Speech-To-Speech Translation System," IEEE Trans. ASLP, vol.14, no. 2 (2006)

\section{THE FUTURE}

Figure 7 depicts the history of speech translation to this point, as well as future research and development prospects. A worldwide research collaboration working on Asian languages hopes to prototype spoken translation via the Internet in 2010. By roughly 2015, the worldwide research collaboration is expected to release a prototype that includes Western European languages and has more standardized interfaces. After many field tests, Japan's Project to Accelerate Benefits to Society (detailed in the next section) expects to build technology for networked voice translation by 2012. Speech translation competence of continuous simultaneous interpretation of business and lectures is predicted to be ready by approximately 2015, and by 2025, it is expected to be available. Multilingual simultaneous interpretation with contextual information and summary is projected to become accessible, moving closer to the goal of simultaneous interpretation.

Speech translation has become more practicable as a result of technological advancement in speech and language research, especially for basic themes with a clear value of use. Speech translation, on the other hand, has only recently progressed to the stage of developing core technologies. Research and development should be accelerated in order to achieve more sophisticated speech translation. The following are some points that should be the focus of future attention. To begin with, corpus-based solutions have the distinguishing property of improving with use. As a result, securing opportunities for field and social testing, and making active use of technologies that have been developed, is important.

Multilingual speech-translation technology can be tested during activities like the Olympics and World Fairs when speakers of many various languages are anticipated to participate. As a result, it is vital to seize these opportunities to advance technology. NICT ran a monitor study at the Beijing Olympics, focusing on Japanese visitors. It created a speech translation system in Beijing that supported proper nouns, and it had monitors utilize speech translation devices to speak throughout the city, utilizing the devices for transit, tourism, and shopping. Users' satisfaction with the service was surveyed using a questionnaire, bringing voice translation technology one step closer to practical implementation.

If Japan wants to want to be a major tourist destination, providing continuous tourism information services to international tourists through spoken language translation might be an efficient method. Meanwhile, as the majority of international residents and employees in Japan grow, 


\section{International Journal of Computer Applications Technology and Research \\ Volume 11-Issue 03, 36-55, 2022, ISSN:-2319-8656 \\ DOI:10.7753/IJCATR1103.1001}

multilingual voice translation may become a necessary tool for local authorities, medical institutions, law enforcement, and schooling. It should assist cut interpretation expenses even if translators are present. However, if all of these methods are employed independently, only partial knowledge would be gathered, making feedback for Research and development wasteful. To enhance efficiency, national and local government, as well as the business sector, will almost certainly need to develop a cooperative framework. Distributing compact translation gadgets to public organizations where their use is anticipated, for example, maybe useful, as could lending these gadgets to foreign workers and visitors for free. Second, speech translation is a technique that converts spoken words into written words in many languages.

Although interpretation into English is important, it will be much more so if speech translation can function directly between Japanese and the local languages of a variety of nations. As a result, expanding the number of languages supported is critical. When it comes to gathering corpora, there are limits to how far this Research and development can go in Japan alone. A plan for collaboration across nations with a wide range of languages is required; in other words, a system is required to allow diverse countries to collaborate on speech translation, speech, and language research. As a system for collaborative R\&D, establishing worldwide spoken language technology research centers and the likes should provide input from a wide variety of studies into the gathering of speech and dialect data language structure. Third, when several nations begin to explore and create speech translation, standardizing the interfaces that connect these multiple language processing modules will be important. The creation of connecting techniques, data formats, dictionaries, and other such tools must have a standardized mindset in mind. We must prevent a situation in which each nation creates its own system that is incompatible with the others. Japan has improved speech translation technology, and as a result, it may lead other nations in terms of standards. Finally, the copyright must be taken into consideration. Speech and language processing necessitate speech and text corpora, and the amount and quality of these corpora have a significant impact on speech translation performance. As a result, the utilization of news broadcasting companies, newspapers, and the Internet is incredibly successful. The current copyright legislation does not allow for secondary applications like these sorts of corpora. It will be required to amend and manage the legislation to make it more flexible in order to explore and create new technologies. The Cultural Council's Copyright Working Group is presently debating this issue, and a decision will be made soon. After the findings of this study are disclosed, it will be important to rearrange the themes for future full-scale speech translation services and review the response, including service models.

The key goal over the next several decades is to create speech recognition algorithms that are as accurate as human performance. This indicates that for both spontaneous and reading the speech, it is regardless of the surroundings, area, and job. The major priorities will be on enhancing spontaneous speech models (i.e., prosodic characteristics and articulatory models, multi-speaker speech, collecting an acceptable volume of conversational speech, etc. ), as well as modeling and training strategies for multi-environment and multidomain scenarios. Then there'll be the issue of language modeling. Several dynamic language models are generally recognized to function better in particular domains. Establishing a language model that functions effectively across several domains will be a significant step toward emulating human performance. The project aims to achieve a very fast dynamic adaptability at the word/sentence level. Finally, the continual improvement of computer efficiency over time, the autonomy from vocabulary, and the engagement of all possible scholars in the area, not just a few universities, will be further elements pushing advancement. The two most important needs for increasing S2ST performance are to improve conversational speech performance and to introduce highly dynamic language models. This is perhaps the most crucial aspect because speaking at less than $10 \%$ in conversational discourse appears to be a difficult difficulty nowadays.

\section{CONCLUSION}




\section{International Journal of Computer Applications Technology and Research \\ Volume 11-Issue 03, 36-55, 2022, ISSN:-2319-8656 \\ DOI:10.7753/IJCATR1103.1001}

Propels in speech and language research have brought Translation of speech near the pragmatic level for basic points where there is a generally clear worth of utilization. At the current level, nonetheless, speech translation has just arrived at the phase of making the center innovations. To accomplish more modern speech translation, innovative work ought to be additionally sped up. The following are a few focuses that should be the subject of center moving advances.

First and foremost, one unmistakable component of corpusbased advances is that they improve with use. It is subsequently crucial to get open doors for field and social testing and to effectively utilize created innovations. Occasions like the Olympics and World Fairs, where speakers of a wide range of dialects can be anticipated to join in, are an optimal chance to handle test multilingual speech-translation technology. It is subsequently indispensable to use these potential chances to propel innovation. NICT directed a screen try at the Beijing Olympics, mostly focusing on explorers from Japan. It fostered a speech translation framework supporting formal people, places, or things in the city of Beijing and had screens use discourse interpretation gadgets to impart in the city, involving the gadgets for such purposes as transportation, touring, and shopping.

Besides, speech translation is an innovation that deciphers communicated words in various languages. Despite the fact that interpretation into English is obviously fundamental, it will likewise be profoundly huge assuming speech translation can work straightforwardly between various languages of the world. Therefore, it is indispensable to expand the number of languages upheld. A plan of a joint effort between nations with a wide range of languages is required; all in all, a component is expected to empower different nations to work in an organization to explore speech translation, speech, and language. Making global communication in language innovation research focuses and so forth as a plan for cooperative Research and development should deliver input from a wide scope of examination into the assortment of discourse and tongue information, language structure, and such.

Thirdly, when numerous nations start to really investigate and foster speech translation, it will be important to normalize the points of interaction to associate these different language handling modules. The improvement of association strategies, information arrangements, word references, and such should keep an eye toward normalization. We should keep away from a circumstance wherein every nation fosters its own framework, and the frameworks are not viable together. Speech translation technology is progressed in such nations like Japan, and these nations can, in this manner, lead different nations with connection to normalization.

At long last, consideration should be given to copyright. Speech and language handling require speech and text corpora, and the presentation of speech translation relies intensely upon the amount and nature of these corpora. Thusly, the utilization of corpora of information broadcasts, papers, and the Web is very successful. Current intellectual property regulation doesn't consider auxiliary uses like these sorts of corpora. To investigate and foster new advances, it will be important to reexamine and manage the law with the goal that it is more flexible.

\section{REFERENCES}

1. Arora, K., Arora, S., and Roy, M.K. 2013. Speech to speech translation: a communication boon. CSIT 1, 207-213. https://doi.org/10.1007/s40012-013-0014-4.

2. Satoshi, N. 2008. Overcoming the Language Barrier with Speech Translation Technology.

3. Mahak, D., and Sumanlata, G. 2015. Speech-to-Speech Translation: A Review. International Journal of Computer Applications, 129 - 13.

4. Satoshi, N., Konstantin, M., Hiromi, N., Genichiro, K., Hisashi, K., Takatoshi, J., Jin-Song, Z., Hirofumi, Y., Eiichiro, S., and Seiichi, Y. 2006. The ATR Multilingual Speech-to-Speech Translation System. IEEE Transactions on Audio, Speech, and Language Processing, 14-2.

5. Gianni, L. 2002. The VI framework program in Europe: Some thoughts about Speech to Speech Translation research.

6. Marija, O., and Martina, L. 2021. Assessing Speech-toSpeech Translation Quality: Case Study of the ILA S2S APP.

7. Lavie, A., Waibel, A., Levin, L., Finke, M., Gates, D., Gavalda, M., Zeppenfeld, T., and Zhan, P. 1997. JANUS 


\section{International Journal of Computer Applications Technology and Research}

Volume 11-Issue 03, 36-55, 2022, ISSN:-2319-8656

DOI:10.7753/IJCATR1103.1001

III: Speech-to-speech translation in multiple languages. In 1997 IEEE International Conference on Acoustics, Speech, and Signal Processing, 99-102. IEEE.

8. Nakamura, S., Markov, K., Nakaiwa, H., Kikui, G.-i., Kawai, H., Jitsuhiro, T., Zhang, J.-S., Yamamoto, H., Sumita, E., and Yamamoto, S., 2006. The ATR multilingual speech-to-speech translation system. IEEE Transactions on Audio, Speech, and Language Processing 14(2), 365-376.

9. Wahlster, W. 2013. Verbmobil: foundations of speechto-speech translation. Springer Science \& Business Media.

10. Jia, Y. Weiss, R. J., Biadsy, F., Macherey, W., Johnson, M., Chen, Z., and Wu, Y. 2019. Direct speech-to-speech trans-lation with a sequence-to-sequence model.

11. Dunbar, E., Cao, X. N., Benjumea, J., Karadayi, J., Bernard, M., Besacier, L., Anguera, X., and Dupoux, E. 2017. The zero resource speech challenge 2017. In 2017 IEEE Automatic Speech Recognition and Understanding Workshop (ASRU), 323-330. IEEE.

12. Jerneja, Ž. G., and Mario Ž. 2006. The VoiceTRAN Speech-to-Speech Translation Communicator. Proceedings of the 5th WSEAS International Conference on Applications of Electrical Engineering, Prague, Czech Republic, 79-83. 